\title{
A functional S-dual in a strong shape category
}

\author{
by
}

Friedrich W. B a u er (Frankfurt a.M.)

\begin{abstract}
In the S-category $\mathfrak{P}$ (with compact-open strong shape mappings, cf. $\S 1$, instead of continuous mappings, and arbitrary finite-dimensional separable metrizable spaces instead of finite polyhedra) there exists according to [1], [2] an S-duality. The S-dual $D \mathbf{X}, \mathbf{X}=(X, n) \in \mathfrak{P}$, turns out to be of the same weak homotopy type as an appropriately defined functional dual $\overline{\left(\mathbf{S}^{0}\right)^{\mathbf{X}}}$ (Corollary 4.9). Sometimes the functional object $\overline{X^{Y}}$ is of the same weak homotopy type as the "real" function space $X^{Y}(\S 5)$.
\end{abstract}

0. Introduction. S-duality occurs in the S-category $\mathfrak{P}$, having pairs $\mathbf{X}=(X, n), X$ a separable metrizable finite-dimensional space, $n \in \mathbb{Z}$, as objects, with stable homotopy classes of so-called coss-morphisms (= strong shape morphisms with compact-open carrier, cf. $[1, \S 2],[2, \S 1])$ as morphisms. The main result is the existence of an S-dual $D \mathbf{X}$, displaying all properties to be expected from classical S-duality (cf. [1, Theorem 4.3], [2, Theorem 2.1]).

One of the basic achievements of classical S-duality lies in the possibility of exhibiting $D \mathbf{X}$, up to weak homotopy equivalence, as a functional object $F\left(\mathbf{X}, \mathbf{S}^{0}\right)([4$, Theorem 3.8] and [7]).

In the present strong-shape-theoretic approach to S-duality (which can be administered to any $X \subset S^{n}$, not only to finite polyhedra) we have, in order to detect functional objects, to follow the same lines which led to "virtual spaces" $X \bar{\wedge} Y$ in $[2, \S 1]$ (which are only under additional assumptions equivalent to the $\wedge$-product $X \wedge Y$ ) and establish "virtual function spaces" $\overline{X^{Y}}(\S 3)$. It turns out $(\S 5)$ that they are sometimes of the same weak homotopy type as the function spaces $X^{Y}$. In general they are, like $X \pi Y$ in $[2, \S 1]$, new objects, which are defined by introducing new coss-morphisms $Z \rightarrow \overline{X^{Y}}(\S 2, \S 3)$.

1991 Mathematics Subject Classification: Primary 55P25, 55P55; Secondary 55N20, $55 \mathrm{M} 05,55 \mathrm{C} 40$.

Key words and phrases: S-duality, functional S-dual, virtual spaces, weak homotopy type, compact-open strong shape. 
The stable functional object $\mathbf{Y}^{\mathbf{Z}}, \mathbf{Y}=(Y, m), \mathbf{Z}=(Z, n)$, resp. $\overline{\mathbf{Y}^{\mathbf{Z}}}$ can be defined as $\left(Y^{Z}, m-n\right)$ resp. $\left(\overline{Y^{Z}}, m-n\right)$. Although $\mathbf{Y}^{\mathbf{Z}}$ is not an object of the original $\mathfrak{P}$ (because $Y^{Z}$ is generally neither separable metric nor finite-dimensional) it can nevertheless be realized as an object of an amended S-category. As a result there is no need to deal with the more involved theory of spectra.

In $\S 1$ we recall some facts from [1], [2] without proofs and set up the notations and conventions used in the sequel. While the next section has only auxiliary character, we introduce in $\S 3$ functional objects (in an amended category coss) and verify a very general exponential law (Theorem 3.4).

The concept of a weak homotopy equivalence, introduced in Definition 4.1, is more restrictive than usual: It turns out that real spaces (as opposed to virtual spaces) are of the same homotopy type iff they are of the same weak homotopy type.

Theorem 4.8 and Corollary 4.9 confirm the existence of a weak homotopy equivalence between the S-dual $D \mathbf{Z}$ of an object $\mathbf{Z}=(Z, m) \in \mathfrak{P}$ and the

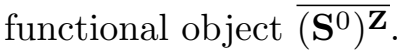

As pointed out already, we set up in $\S 5$ a relationship between the virtual space $\overline{Y^{Z}}$ and the real space $Y^{Z}$ (although not an object of Met, the category of separable metrizable spaces). In particular, the classical presentation of an S-dual of a finite polyhedron by means of function spaces (Theorem 4.8 of [4]) is confirmed in our framework (Corollary 5.4).

We resume all notations and results of [1] and [2] without further mention. Details of constructions and proofs (e.g. those of new coss-morphisms) will be omitted whenever they coincide with those in [1], [2] concerning related concepts or assertions.

1. Preliminaries. In $[1, \S 2]$, we introduced the concept of cossmorphisms (= compact-open strong shape morphisms or strong shape morphisms with compact-open support) $\alpha: X \rightarrow Y$ between spaces $X, Y \in$ Met (= category of separable metrizable spaces and (based or unbased) continuous mappings). Such an $\alpha$ assigns, roughly speaking, to each compact subset $X^{\prime} \subset X$ a strong shape morphism $X^{\prime} \rightarrow Y^{\prime} \subset Y$ with $Y^{\prime}$ compact, and $\alpha$ is supposed to behave on ANRs $Q \supset Y$ like an ordinary (stable) shape morphism. So $\operatorname{coss}(X, Y)$ appears as some kind of double limit (cf. Definition 2.1 of [1]).

In a next step we used these coss-morphisms to set up the S-category $\mathfrak{P}$ with pairs $\mathbf{X}=(X, n), X \in \mathbf{M e t}, n \in \mathbb{Z}$, as objects and stable homotopy classes of coss-morphisms as morphisms. This allowed us to establish for each $\mathbf{X}=(X, n) \in \mathfrak{P}, X$ finite-dimensional in Met, an S-dual $D \mathbf{X}$ satisfying $D^{2} \mathbf{X} \approx \mathbf{X}$ and $\{\mathbf{X}, \mathbf{Y}\}_{c} \approx\{D \mathbf{Y}, D \mathbf{X}\}_{c}$. 
In $[2, \S 2]$ we amended the category coss by adding new morphisms $X \rightarrow$ $X \bar{\wedge} Z$ and $X \bar{\wedge} Y \rightarrow Z$, where $Y \bar{\wedge} Z$ and $X \bar{\wedge} Y$ appear either as pairs of spaces in Met or alternatively as new objects (so-called "virtual spaces"). Under certain assumptions, these virtual spaces are associated with $\wedge$-products $Y \wedge Z$ and $X \wedge Y$ (cf. [2, Proposition 1.2]).

For all details we refer to [1] and [2] without further explanation.

In the present paper we need 1) in addition to separable metric spaces $Y, Z$ their function spaces $\left.Y^{Z}, 2\right)$ new objects $\overline{Y^{Z}}$ which can again be regarded either simply as pairs of spaces or alternatively as new virtual spaces. They are determined by means of new coss-morphisms (cf. §3) $X \rightarrow \overline{Y^{Z}}, X, Y, Z \in$ Met. Again under certain assumptions, they are associated with the "real" function space $Y^{Z}(\S 5)$.

In order to ensure the validity of the classical exponential law ([5, Ch. XII]) we have to restrict the class of ANRs to locally compact ANRs, calling these spaces l-ANRs (or simply "good spaces" or "good spaces in Met"). This is not very restrictive, because according to [1, Lemma 2.6] we are allowed to confine ourselves anyway to open subsets of some $S^{n}$ or some Hilbert cube (which are always locally compact). All ANRs $P$ appearing as exponents in some $X^{P}$ are always assumed to be l-ANRs.

In addition, we still have arbitrary ANRs, e.g. those of the form $P^{Z}, P$ any ANR, $Z \in$ Met compact. Even for $P \in \mathbf{l}$-ANR, $Z \in$ Met compact, $P^{Z}$ is in general neither in Met nor locally compact. These ANRs (which are not necessarily l-ANRs) are needed for establishing strong shape morphisms $\bar{a}: X \rightarrow \overline{Y^{Z}}$ :

Recall (e.g. [3]) that a strong shape morphism $\bar{f}: A \rightarrow B, A, B$ compact metric, is a 2-functor $\bar{f}: \mathbf{P}_{B} \rightarrow \mathbf{P}_{A}$ between two 2-categories associated with the respective spaces. The objects of $\mathbf{P}_{A}$ are continuous mappings $g: A \rightarrow P \in \mathbf{A N R}$, the 1-morphisms $(r, \omega): g_{1} \rightarrow g_{2}$, where $g_{i}: A \rightarrow P_{i}$, are pairs with $r: P_{1} \rightarrow P_{2}$ continuous and $\omega: r g_{1} \simeq g_{2}$ a homotopy, while the 2-morphisms are pairs $(\omega, \xi)$ with $\omega$ a homotopy and $\xi$ a 2-homotopy.

Although this is needed only for compacta or good spaces, the category $\mathbf{P}_{A}$ can be established for any space $A$. There exists a well-developed, but much more involved theory of strong shape for arbitrary spaces, which works with $\infty$-categories (or some simplicial equivalent) $\mathbf{P}_{A}$ and $\infty$-functors (cf. [6] for further references), but we do not need this theory in the course of this paper.

In order to establish $\bar{a}: X \rightarrow \overline{Y^{Z}}$ for $X$ compact or good, and $Y, Z$ compacta, we simply employ the 2-subcategory $\left(\mathbf{P}_{Y}\right)^{Z} \subset \mathbf{P}_{Y^{Z}}$ consisting of all objects $g^{Z}: Y^{Z} \rightarrow P^{Z}, g \in \mathbf{P}_{Y}$, and correspondingly for the 1- and 2-morphisms (i.e. the image category of the functor ()$^{Z}: \mathbf{P}_{Y} \rightarrow \mathbf{P}_{Y^{Z}}$ ).

If $X$ is a good space, we have in $\mathbf{P}_{X}$ an initial object $1: X \rightarrow X$. 
This is the only point where one needs some information about strong shape morphisms. Although in $\S 3$ we define coss-morphisms $X \rightarrow \overline{Y^{Z}}$ for any $X, Y, Z \in$ Met we do not need strong shape theory for spaces other than compacta and good spaces.

2. Functional objects in a strong shape category. For $Y, Z \in$ Met, $Y^{Z}$ denotes the function space with the compact-open topology. In analogy to the construction of $Y \bar{\wedge} Z$ in $[2, \S 1]$ the virtual function space $\bar{Y}^{Z}$ is defined by setting up new shape morphisms $\bar{a}: X \rightarrow \overline{Y^{Z}}, X \in$ Met. This will be finally accomplished in $\S 3$. In this section we deal with the special case of $X$ compact or good (i.e. l-ANR) while $Y, Z \in$ Met either compact; or $Y$ good and $Z$ compact; or $Y$ and $Z$ good.

In all these cases a morphism $\bar{a}: X \rightarrow \overline{Y^{Z}}$ is a 2-functor $\left(\mathbf{P}_{Y}\right)^{Z} \rightarrow \mathbf{P}_{X}$ such that $\bar{a}\left(g^{Z}\right): X \rightarrow P^{Z}$ for $P \in \mathbf{A N R}$ and $(g: Y \rightarrow P) \in$ ob $\mathbf{P}_{Y}$, and correspondingly for 1- and 2-morphisms.

Observe that $P^{Z}$ with $Z$ compact is an ANR (although not necessarily an l-ANR).

If $Z$ is compact and $Y$ good, we have $g=1_{Y}: Y \rightarrow Y$ and therefore $\bar{a}\left(1_{Y}^{Z}\right): X \rightarrow Y^{Z}$.

It is possible to define homotopy classes of individual mappings $\bar{a}$ so defined. In order to set up stable homotopy classes we define

$$
\Sigma\left(\overline{Y^{Z}}\right)=\overline{(\Sigma Y)^{Z}}
$$

and introduce for each $\bar{a}: X \rightarrow \overline{Y^{Z}}$ a mapping $\Sigma \bar{a}: \Sigma X \rightarrow \Sigma\left(\overline{Y^{Z}}\right)$ in the following way:

For $(g: \Sigma Y \rightarrow P) \in \mathbf{P}_{\Sigma Y}$ we have $(\widetilde{g}: Y \rightarrow \Omega P) \in \mathbf{P}_{Y}, \bar{a}(\widetilde{g}):$ $X \rightarrow(\Omega P)^{Z}$ and by taking adjoints we obtain successively $X \wedge Z \rightarrow \Omega P$, $\Sigma X \wedge Z \rightarrow P$ and finally the required $(\Sigma \bar{a})(g): \Sigma X \rightarrow P^{Z}$.

Now we can stabilize and denote by $\overline{\left\{X, \overline{Y^{Z}}\right\}}$ the set of stable homotopy classes $\{\bar{a}\}$ of mappings $\bar{a}: X \rightarrow \overline{Y^{Z}}$, whenever these are defined.

Suspension induces now an isomorphism

$$
\Sigma_{\star}: \overline{\left\{X, \overline{Y^{Z}}\right\}} \stackrel{\approx}{\rightarrow\left\{\Sigma X, \overline{(\Sigma Y)^{Z}}\right\}},
$$

provided both sides are defined.

In addition to strong shape morphisms $\bar{a}: X \rightarrow \overline{Y^{Z}}$ we need 1) the induced morphisms

$$
\overline{Y^{Z}} \stackrel{\bar{f}^{Z}}{\longrightarrow} \overline{Y_{1}^{Z}}
$$

for strong shape morphisms $\bar{f}: Y \rightarrow Y_{1}$, defined as a functorial (in $X$ ) mapping

$$
\overline{\left\{X, \overline{Y^{Z}}\right\}} \rightarrow \overline{\left\{X, \overline{Y_{1}^{Z}}\right\}}
$$


by setting

$$
\left(\bar{f}^{Z} \bar{a}\right)\left(g^{\prime Z}: Y_{1}^{Z} \rightarrow P^{Z}\right)=\bar{a}\left(\bar{f}\left(g^{\prime}\right)^{Z}\right)
$$

and 2) the induced morphisms

$$
\overline{Y^{Z}} \stackrel{\overline{Y^{f}}}{\longrightarrow} \overline{Y^{Z}}
$$

for $Y$ good and $f: Z \rightarrow Z_{1}$ continuous. Again

$$
\overline{\left\{X, \overline{Y^{Z_{1}}}\right\}} \rightarrow \overline{\left\{X, \overline{Y^{Z}}\right\}}
$$

is defined by setting

$$
\left(\overline{Y^{f}} \bar{a}\right)(g)=P^{f} \bar{a}(g), \quad(g: Y \rightarrow P) \in \mathbf{P}_{Y} .
$$

We have:

Lemma 2.1. 1) If $f: X \rightarrow Y^{Z}$ is continuous, $Z$ compact or good, and $Y$ compact or good, then $f$ induces a strong shape morphism $\bar{f}: X \rightarrow \overline{Y^{Z}}$.

2) If $\bar{f}: X \rightarrow \overline{Y^{Z}}$ is a strong shape morphism with $Y$ good and $Z$ compact, then $\bar{f}\left(1_{Y}\right): X \rightarrow Y^{Z}$ determines $\bar{f}$ up to homotopy.

Pr o of. The first part is obvious. The second follows by standard arguments in strong shape theory (cf. [3]) because $Y^{Z}$ is an ANR.

To establish an exponential law for coss-morphisms and arbitrary spaces in Met (Theorem 3.4) we need a very modest exponential law for strong shape morphisms:

Lemma 2.2. Suppose that either 1) $X, Y, Z$ are compact or 2) $X, Y, Z$ are good (implying in particular that $Z$ is locally compact). Then there exists a natural bijection

$$
\overline{\left\{X, \overline{Y^{Z}}\right\}} \approx \overline{\{X \wedge Z, Y\}} .
$$

Pro of. In both cases the classical exponential law applies and we obtain a bijection between

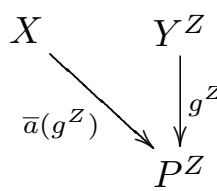

and<smiles>[Y][V][Te][3H]</smiles>

with $\bar{a} \in \overline{\left\{X, \overline{Y^{Z}}\right\}}$ and $(\ldots)^{\prime}$ denoting the adjoint of $(\ldots)$. This establishes a bijection between individual strong shape morphisms $X \rightarrow \overline{Y^{Z}}$ and $X \wedge Z \rightarrow Y$, which, carrying over to stable homotopy classes, furnishes a bijection (1).

As a first application we notice: 
COROLLARY 2.3. Under the same assumptions as in $2.2, \overline{\left\{X, \overline{Y^{Z}}\right\}}$ carries a natural abelian group structure.

Proof. The structure is inherited from $\overline{\{X \wedge Z, Y\}}$, which carries such a structure in both relevant cases.

COROLLARY 2.4. The bijection (1) is an isomorphism of abelian groups.

3. The exponential law. Analogously to $Y \wedge Z$ we now define new coss-morphisms $\alpha: X \rightarrow \overline{Y^{Z}}$ for any $X, Y, Z \in$ Met. To this end we resume the notations of $[2, \S 1]$ concerning the mappings $a: X^{\prime} \rightarrow X, b: Y^{\prime} \rightarrow Y$, $c: Z^{\prime} \rightarrow Z, r: X \rightarrow P, s: Y \rightarrow Q, t: Z \rightarrow R, \widetilde{t}: Z^{\prime} \rightarrow \widetilde{R}$ and $\widetilde{a}: X^{\prime} \rightarrow \widetilde{P}$, with $X^{\prime}, Y^{\prime}, Z^{\prime}$ compact and $P, Q, R, \widetilde{P}, \widetilde{R}$ good.

We consider stably homotopy commutative diagrams $\sigma$ :

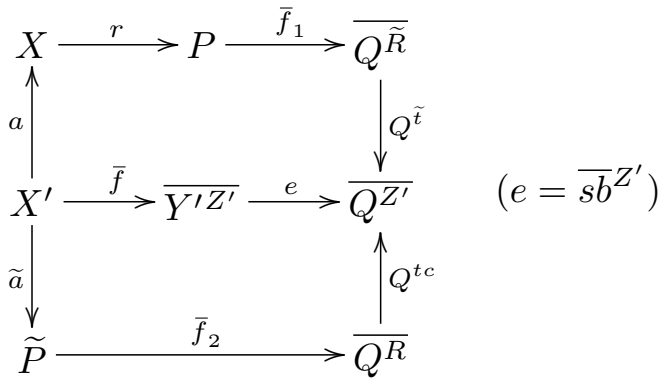

More precisely, all arrows represent either elements in some $\overline{\{\ldots, \ldots\}}$, induced mappings (as defined in $\S 2$ ) or stable homotopy classes of continuous mappings. This concept is of course associated with that of Definition 1.1 in [2]. We denote by $T(a, c, t)$ the class of all such diagrams with fixed $a, c, t$ (of course for given $X, Y, Z$ ). In the same way as in $[2, \S 1]$ or in $[1, \S 2]$ we establish an equivalence relation between elements in $T(a, c, t)$, denoting the set of all equivalence classes by $T[a, c, t]$.

An $\alpha: X \rightarrow \overline{Y^{Z}}$ is (as in $[2, \S 1]$ ) an assignment which assigns to each triple $(a, c, t)$ a class $[\sigma]=\alpha(a, c, t) \in T[a, c, t]$ such that obvious compatibility conditions are satisfied. These compatibility conditions are, of course, associated with those leading to $X \pi Z \rightarrow Y$ in [2, Definition 1.1].

We summarize, using the customary lim notation:

Definition 3.1. For any $X, Y, Z$ in Met we set

$$
\operatorname{coss}\left(X, \overline{Y^{Z}}\right)=\varliminf_{\bigsqcup} \lim T(a, c, t)=\left\{X, \overline{Y^{Z}}\right\}_{c} .
$$

We have induced mappings:

1) Let $\beta \in\left\{Y, Y_{1}\right\}_{c}$ be a coss-morphism between spaces (cf. [1, $\left.\S 2\right]$ ). Then we define $\beta^{Z}: Y^{Z} \rightarrow Y_{1}^{Z}, Z \in$ Met, such that for any $\alpha \in\left\{X, \overline{Y^{Z}}\right\}_{c}$ there is a $\overline{\beta^{Z}} \alpha \in\left\{X, \overline{Y_{1}^{Z}}\right\}_{c}$, in a functorial way: 
Suppose that $\sigma=\beta\left(b, t_{1}\right) \in \in T\left[b, t_{1}\right]$

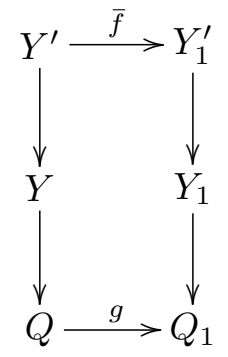

represents $\beta$. Then in the preceding section we have defined

$$
\overline{Y^{\prime Z^{\prime}}} \stackrel{\bar{f}^{Z^{\prime}}}{\longrightarrow} \overline{Y_{1}^{\prime Z^{\prime}}}, \quad \overline{Q^{R}} \stackrel{g^{R}}{\longrightarrow} \overline{Q_{1}^{R}}, \quad \overline{Q^{\widetilde{R}}} \stackrel{g^{\widetilde{R}}}{\longrightarrow} \overline{Q_{1}^{\widetilde{R}}},
$$

and consequently for given $\alpha: X \rightarrow \overline{Y^{Z}}$,

$$
X^{\prime} \rightarrow \overline{Y^{\prime Z}} \rightarrow \overline{Y_{1}^{Z^{\prime}}}, \quad \widetilde{P} \rightarrow \overline{Q^{R}} \rightarrow \overline{Q_{1}^{R}}, \quad P \rightarrow \overline{Q^{\widetilde{R}}} \rightarrow \overline{Q_{1}^{\widetilde{R}}},
$$

furnishing us with a representative $\beta^{Z} \alpha: X \rightarrow Y_{1}^{Z}$.

2) Let $f: Z \rightarrow Z_{1}$ be continuous. Then for given compact $c: Z^{\prime} \rightarrow Z$ we find a $Z_{1}^{\prime}=f c\left(Z^{\prime}\right)$ and an $\left(f \mid c\left(Z^{\prime}\right)\right)=f^{\prime}: Z^{\prime} \rightarrow Z_{1}^{\prime}$. On the other hand, for $t_{1}: Z_{1} \rightarrow R_{1}$ we find an extension $f_{1}: R \rightarrow R_{1}$ of $t_{1} f$ over some good $R \supset Z$ up to homotopy (cf. [1,2.5]).

In the same way we proceed with $\widetilde{t}_{1}: Z_{1}^{\prime} \rightarrow \widetilde{R}_{1}$, getting an $f_{1}: \widetilde{R} \rightarrow \widetilde{R}_{1}$.

As a result we obtain

$$
\overline{Y^{f}}: \overline{Y^{Z_{1}}} \rightarrow \overline{Y^{Z}}
$$

such that for any $\alpha \in\left\{X, \overline{Y^{Z_{1}}}\right\}_{c}$ we detect a $\overline{Y^{f}} \alpha \in\left\{X, \overline{Y^{Z}}\right\}_{c}$ in a functorial way.

3) Let $\gamma: X_{1} \rightarrow X$ be a coss-morphism between spaces. Then we have in an obvious way a composition

$$
X_{1} \stackrel{\gamma}{\rightarrow} X \stackrel{\beta}{\rightarrow} \overline{Y^{Z}}
$$

All these morphisms between spaces (the old coss-morphisms) and between new objects $\overline{Y^{Z}}$ form a category.

In $\S 5$ we will investigate under what circumstances $\overline{Y^{Z}}$ and $Y^{Z}$ are of the same weak homotopy type. This has to be properly defined because in general $Y^{Z}$ is not an object of Met. Here we can already make the following observation:

Lemma 3.2. If $Z=\{1,2, \ldots, n\}$ is a finite space, then there exists a bijection

$$
\left\{X, \overline{Y^{Z}}\right\}_{c} \approx\left\{X, \sum_{1}^{n} Y\right\}_{c}
$$

where $\sum_{1}^{n} Y$ is the free union of $n$ copies of $Y$. 
Proof. Because $Z$ is good and compact, we are always allowed to work with $Z=Z^{\prime}=R=\widetilde{R}$, so that $Y^{\prime Z^{\prime}}=Y^{\prime Z}=\sum_{1}^{n} Y^{\prime}$ and $Q^{R}=Q^{\widetilde{R}}=\sum_{1}^{n} Q$. Let $\alpha: X \rightarrow \overline{Y^{Z}}$ be a coss-morphism. Then we have $X^{\prime} \rightarrow Y^{\prime Z^{\prime}}=\sum_{1}^{n} Y^{\prime}$, $\widetilde{P} \rightarrow \sum_{1}^{n} Q$ and $P \rightarrow \sum_{1}^{n} Q$ as representatives of $\alpha$, allowing us to set $P=\widetilde{P}$. As a result, $\alpha$ is described by diagrams

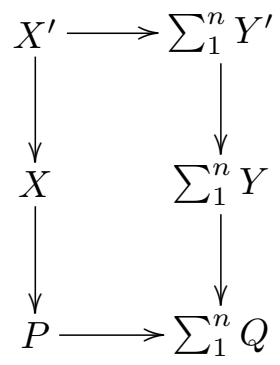

hence by a coss-morphism $X \rightarrow \sum_{1}^{n} Y$ establishing the assignment

$$
\left\{X, \overline{Y^{Z}}\right\}_{c} \rightarrow\left\{X, \sum_{1}^{n} Y\right\}_{c}
$$

This assignment can be easily inverted.

This can be expressed by saying that in this case the virtual space $\overline{Y^{Z}}$ is a real space $\sum_{1}^{n} Y$ in coss.

Definition 3.3. The suspension of a functional object is defined by

$$
\Sigma\left(\overline{Y^{Z}}\right)=\overline{(\Sigma Y)^{Z}} \text {. }
$$

The exponential law in the category coss is the following

Theorem 3.4. For any three spaces $X, Y, Z \in$ Met there exists a natural isomorphism

$$
\eta:\{X \pi Z, Y\}_{c} \approx\left\{X, \overline{Y^{Z}}\right\}_{c} .
$$

Proof. A morphism $\alpha: X \pi Z \rightarrow Y$ determines $\alpha(a, c, t)$, i.e. cossmorphisms $\bar{f}: X^{\prime} \wedge Z^{\prime} \rightarrow Y^{\prime}, f_{1}: P \wedge \widetilde{R} \rightarrow Q$ and $f_{2}: \widetilde{P} \wedge R \rightarrow Q$ satisfying certain compatibility conditions embodied in [2, Definition 1.1]. On the other hand, we can apply 2.1.1) and 2.2 obtaining the associated adjoints

$$
\bar{f}^{\prime}: X^{\prime} \rightarrow \overline{Y^{\prime Z^{\prime}}}, \quad \overline{f_{1}^{\prime}}: P \rightarrow \overline{Q^{\widetilde{R}}}, \quad \overline{f_{2}^{\prime}}: \widetilde{P} \rightarrow \overline{Q^{R}},
$$

where the last two mappings are simply continuous mappings into $Q^{\widetilde{R}}$ resp. $Q^{R}$, satisfying (1).

All l-ANRs are supposed to be locally compact, hence the exponential law (Lemma 2.2) applies. 
This establishes an assignment

$$
\eta:\{X \wedge Z, Y\}_{c} \rightarrow\left\{X, \overline{Y^{Z}}\right\}_{c}
$$

which is of course natural (with respect to coss-morphisms between spaces $X \rightarrow X_{1}^{\prime}, Y \rightarrow Y_{1}$, and continuous $Z \rightarrow Z_{1}$ ). We obtain an inverse $\eta^{-1}$ by reversing the procedure leading to $\eta$. This completes the proof.

As a first application we can use [2, Proposition 1.7] in order to establish a stability property of the new $\{\ldots, \ldots\}_{c}$ sets:

COROLlary 3.5. There exists a natural isomorphism

$$
\left\{\Sigma X, \Sigma\left(\overline{Y^{Z}}\right)\right\}_{c} \approx\left\{X, \overline{Y^{Z}}\right\}_{c} .
$$

Proof. We have the following chain of natural equivalences and identities:

$$
\begin{aligned}
\left\{\Sigma X, \Sigma\left(\overline{Y^{Z}}\right)\right\}_{c} & \approx\left\{\Sigma X, \overline{(\Sigma Y)^{Z}}\right\}_{c} \approx\{\Sigma X \Uparrow Z, \Sigma Y\}_{c} \\
& \approx\{X \pi Z, Y\}_{c} \approx\left\{X, \overline{Z^{Y}}\right\}_{c} .
\end{aligned}
$$

Since $\{X \pi Z, Y\}_{c}$ always carries a natural abelian group structure (cf. $\left[2\right.$, Proposition 1.8]) the same holds for $\left\{X, \overline{Y^{Z}}\right\}_{c}$ and we have

COROLlaRY 3.6. $\left\{X, \overline{Y^{Z}}\right\}_{c}$ carries a natural abelian group structure such that $\eta$ in 3.4 becomes a natural isomorphism.

Remarks. 1) If $X, Y, Z$ satisfy the requirements of $\S 2$, then

$$
\left\{X, \overline{Y^{Z}}\right\}_{c}=\overline{\left\{X, \overline{Y^{Z}}\right\}} \text {. }
$$

2) Definition 3.3 is the reason that, in contrast to function spaces $Y^{Z}$, there is no difference between $\Sigma\left(\overline{Y^{Z}}\right)$ and $\overline{(\Sigma Y)^{Z}}$.

3) Another version of virtual function spaces together with an exponential law has (in a different context and for different purposes) been the subject of [8].

4. Weak homotopy equivalences. Suppose $A$ and $B$ are spaces or virtual spaces. Then we define:

Definition 4.1. $A$ and $B$ are of the same weak homotopy type (in symbols: $A \simeq B$ ) whenever there exists a natural equivalence

$$
\omega:\{X, A\}_{c} \approx\{X, B\}_{c}, \quad X \in \text { Met. }
$$

Naturality means that $\omega$ commutes with all homomorphisms induced by coss-morphisms between spaces $X$.

If $A$ or $B$ is a space, we can say more:

Lemma 4.2. Suppose 1) $A$ is a space, and 2) $A$ and $B$ are of the same weak homotopy type. Then there exists a coss-morphism $\alpha: A \rightarrow B$ such 
that

$$
\alpha^{*}:\{X, B\}_{c} \stackrel{\widetilde{\sim}}{\rightarrow}\{X, A\}_{c}
$$

is an isomorphism for all spaces $X$, hence $\omega^{-1}$ in (1) is induced by a cossmorphism.

P r o of. The classical argument of the Yoneda lemma applies: We insert $X=A$ and detect

$$
\alpha=\omega\left(1_{A}\right): A \rightarrow B .
$$

The verification that $\alpha^{*}=\omega^{-1}$ is now standard.

If $A$ and $B$ are spaces, then weak and ordinary homotopy types coincide:

Lemma 4.3. If $A$ and $B$ are both spaces, then $A$ and $B$ are of the same weak homotopy type whenever they are of the same homotopy type (i.e. iff there exist coss-morphisms $\alpha: A \rightarrow B$ and $\beta: B \rightarrow A$ such that $\alpha \beta=1_{B}$ and $\left.\beta \alpha=1_{A}\right)$.

Proof. $A \simeq B$ implies the existence of $\alpha$ and $\beta$ because of 4.2. The other direction is trivial.

LEMMA 4.4. Being of the same weak homotopy type is an equivalence relation.

The proof is immediate.

LEMmA 4.5. There exists a weak homotopy equivalence

$$
\overline{Y^{Z}} \simeq \Sigma\left(\overline{Y^{\Sigma Z}}\right) .
$$

Proof. There are natural equivalences (applying $[2,1.7]$ )

$$
\begin{aligned}
\left\{X, \Sigma\left(\overline{Y^{\Sigma Z}}\right)\right\}_{c} & \approx\left\{X, \overline{(\Sigma Y)^{\Sigma Z}}\right\}_{c} \approx\{X \wedge \Sigma Z, \Sigma Y\}_{c} \\
& \approx\{\Sigma X \wedge Z, \Sigma Y\}_{c} \approx\{X \wedge Z, Y\}_{c} \approx\left\{X, \overline{Y^{Z}}\right\}_{c} .
\end{aligned}
$$

This enables us to define functional objects in the S-category $\mathfrak{P}$ (cf. $[1$, $\S 4])$ by setting

$$
\overline{(Y, m)^{(Z, n)}}=\left(\overline{Y^{Z}}, m-n\right) .
$$

This is in accordance with the convention (cf. $[1, \S 4(1)]$ )

$$
(\Sigma X, m)=(X, m+1) .
$$

By a space $\mathbf{X} \in \mathfrak{P}$ we mean an $\mathbf{X}=(X, n), X \in$ Met. Moreover, we retain the convention to write $X$ for $(X, 0)$.

We can now extend Definition 4.1 over the category $\mathfrak{P}$ :

Definition 4.1'. Two objects $\mathbf{A}, \mathbf{B} \in \mathfrak{P}$ are of the same weak homotopy type $(\mathbf{A} \simeq \mathbf{B})$ whenever there exists a natural (in $X$ ) equivalence

$$
\omega:\{\mathbf{X}, \mathbf{A}\}_{c} \approx\{\mathbf{X}, \mathbf{B}\}_{c}, \quad \mathbf{X} \in \mathfrak{P} \text { a space. }
$$


Moreover, the exponential law 3.4 extends over $\mathfrak{P}$ :

Proposition 4.6. For any three spaces $\mathbf{X}, \mathbf{Y}, \mathbf{Z} \in \mathfrak{P}$ we have a natural equivalence

$$
\eta:\{\mathbf{X} \wedge \mathbf{Y}, \mathbf{Z}\}_{c} \approx\left\{\mathbf{X}, \overline{\mathbf{Z}^{\mathbf{Y}}}\right\}_{c}
$$

Lemma 4.5 implies

Lemma $4.5^{\prime}$. Let $\mathbf{X}, \mathbf{Y}, \mathbf{Z}$ be spaces. Then we have a weak homotopy equivalence

$$
\Sigma^{-1}\left(\overline{\mathbf{Y}^{\mathbf{Z}}}\right) \simeq \overline{\mathbf{Y}^{\Sigma \mathbf{Z}}}
$$

Returning to spaces in Met we have:

Theorem 4.7. Let $Y \in$ Met be any space and $n$ an integer such that $D_{n} Y$ is geometrically defined. Then $D_{n} Y$ and $\overline{S^{n Y}}$ are of the same weak homotopy type.

Proof. From 3.4 and [2, Theorem 2.1] we deduce natural equivalences

$$
\begin{aligned}
& \left\{X \bar{\wedge}, S^{n}\right\}_{c} \approx\left\{X, \overline{S^{n Y}}\right\}_{c}, \\
& \left\{X \wedge Y, S^{n}\right\}_{c} \approx\left\{X, D Y \wedge S^{n}\right\}_{c} \approx\left\{X, D_{n} Y\right\}_{c}
\end{aligned}
$$

(observe that we have $D_{n} Y=\Sigma^{n}\left(D_{0} Y\right)$ because of $[1, \S 4(2)]$ ), so that $\overline{S^{n Y}} \simeq D_{n} Y$ follows.

In the category $\mathfrak{P}$ we can say a little more:

TheOREM 4.8. For any two $\mathbf{Y}, \mathbf{Z} \in \mathfrak{P}$, where $\mathbf{Y}=(Y, m), \mathbf{Z}=(Z, n)$ (i.e. $\mathbf{Y}, \mathbf{Z}$ are "spaces"), we have a weak homotopy equivalence

$$
\overline{\mathbf{Y}^{\mathbf{Z}}} \simeq D \mathbf{Z} \wedge \mathbf{Y} .
$$

Proof. As in the proof of 4.7 we have

$$
\{\mathbf{X} \wedge \mathbf{Z}, \mathbf{Y}\}_{c} \approx\{\mathbf{X}, \overline{\mathbf{Y}}\}_{c}, \quad\{\mathbf{X} \wedge \mathbf{Z}, \mathbf{Y}\}_{c} \approx\{\mathbf{X}, D \mathbf{Z} \pi \mathbf{Y}\}_{c}
$$

implying the assertion in view of Definition $4.1^{\prime}$.

The dual $D Y$ of any object in $\mathfrak{P}$ can be expressed, up to weak homotopy equivalence, by a functional object:

Corollary 4.9. For any space $\mathbf{Y} \in \mathfrak{P}$ we have

$$
D \mathbf{Y} \simeq \overline{\left(\mathbf{S}^{0}\right)^{\mathbf{Y}}} .
$$

Remarks. 1) If we agree (as in the remark at the end of $[2, \S 1]$ ) to consider $X \wedge Y$ as a virtual space, then $[2,1.2(4)]$ means that under certain conditions $X \wedge Y$ and $X \wedge Y$ (i.e. a real and a virtual space) are of the same weak homotopy type. We are going to implement the same kind of procedure to the virtual space $\overline{Y^{Z}}$ in the following section.

2) Because of 4.5 and (2) we get along with the much less involved S-category $\mathfrak{P}$ rather than a category of spectra (cf. [4]). 
5. The relationship between $Y^{Z}$ and $\overline{Y^{Z}}$. While $\overline{Y^{Z}}$ is a virtual space in coss, $Y^{Z}$ is a real space, which is in general not an object of Met, hence not contained in coss. Therefore, in order to compare these two objects, we have to implement concepts which are not inherent in the category coss.

Denote by $\left\{X, Y^{Z}\right\}$ the set of all stable homotopy classes of continuous mappings, carrying in a well-known way an abelian group structure. Here stable has to be interpreted in the sense of $\S 2, \S 3: a_{1}: \Sigma^{k} X \rightarrow\left(\Sigma^{k} Y\right)^{Z}$ and $a_{2}: \Sigma^{l} X \rightarrow\left(\Sigma^{l} Y\right)^{Z}$ are stably homotopic (i.e. equivalent) whenever $a_{1}^{\prime}: \Sigma^{k} X \wedge Z \rightarrow \Sigma^{k} Y$ and $a_{2}^{\prime}: \Sigma^{l} X \wedge Z \rightarrow \Sigma^{l} Y$ are stably homotopic (in the usual sense). Since $\Sigma Y^{Z}$ and $(\Sigma Y)^{Z}$ are not homotopy equivalent, this is not the same as the ordinary concept of stable homotopy if function spaces as targets are involved.

Theorem 5.1. 1) If $Y$ is good and $Z$ compact, then for compact $X$ there exists a natural isomorphism of abelian groups

$$
\omega:\left\{X, \overline{Y^{Z}}\right\}_{c} \simeq\left\{X, Y^{Z}\right\} .
$$

2) If in addition $Z$ is also good, then we find a natural isomorphism $\omega$ for any $X \in$ Met.

Naturality means of course that $\omega$ commutes with homomorphisms induced by coss-morphisms $\alpha: X \rightarrow X_{1}$ between spaces.

Proof. Firstly, for any $X$, good $Y$ and compact $Z$ we establish a natural homomorphism

$$
\omega:\left\{X, \overline{Y^{Z}}\right\}_{c} \rightarrow\left\{X, Y^{Z}\right\}
$$

and investigate under what additional assumptions $\omega$ becomes an isomorphism.

Let $\alpha: X \rightarrow \overline{Y^{Z}}$ be given, and $a: X^{\prime} \rightarrow X$ and $b: Y^{\prime} \rightarrow Y$ be continuous, with $X^{\prime}, Y^{\prime}$ compact. Then we find a strong shape morphism $X^{\prime} \rightarrow \overline{Y^{\prime Z}}$ as well as continuous mappings

$$
P \rightarrow Y^{\widetilde{R}}, \quad \widetilde{P} \rightarrow Y^{R},
$$

observing that we can assume $Z=Z^{\prime}, Q=Y$ and $R=\widetilde{R}$. So we detect a continuous map

$$
X \stackrel{a}{\rightarrow} Y^{\widetilde{R}}=Y^{R} \stackrel{Y^{t}}{\rightarrow} Y^{Z} .
$$

This yields a natural homomorphism

$$
\omega:\left\{X, \overline{Y^{Z}}\right\}_{c} \rightarrow\left\{X, Y^{Z}\right\} .
$$

Suppose now that $f: X \rightarrow Y^{Z}$ is continuous and $a: X^{\prime} \rightarrow X$ with $X^{\prime}$ compact as before. Then we have the adjoint $(f a)^{\prime}: X^{\prime} \wedge Z \rightarrow Y$ and good $\widetilde{P}, R$ over which $(f a)^{\prime}$ extends:

$$
\widetilde{f^{\prime}}: \widetilde{P} \wedge R \rightarrow Y
$$


hence

$$
\widetilde{f}: \widetilde{P} \rightarrow Y^{R}
$$

Moreover, since $X^{\prime} \wedge Z$ is compact, there exists a compact subset $Y^{\prime} \subset Y$ through which $(f a)^{\prime}$ factorizes. The adjoint is a continuous map $X^{\prime} \rightarrow Y^{\prime Z}$, giving rise to a strong shape morphism $X^{\prime} \rightarrow \overline{Y^{\prime Z}}$.

1) Now suppose that $X$ is compact. Then we can argue about $X \wedge Z \rightarrow Y$ as we did for $X^{\prime} \wedge Z \rightarrow Y$ and find some good $P, R$ and a $P \wedge R \rightarrow Y$ and its adjoint $P \rightarrow Y^{R}=Y^{\widetilde{R}}$.

2) If $X$ is arbitrary but $Z$ good, we are allowed to set $R=\widetilde{R}=Z$ and, because $Y^{Z}$ is good, extend $f$ over some good $P$ up to homotopy (cf. [1, 2.5]), establishing an $\widetilde{f}: P \rightarrow Y^{Z}=Y^{\widetilde{R}}$.

This provides us in both cases with a $\mu(f)=\alpha: X \rightarrow \overline{Y^{Z}}$ such that $\omega(\alpha)=\{f\}$ and $\mu \omega(\alpha)=\alpha$ holds.

Theorem 5.1. allows of course a reformulation in the S-category $\mathfrak{P}$ and in the S-category $\mathfrak{P}^{\prime}$ (using continuous instead of coss-morphisms).

Recall that $\mathbf{X}=(X, m)$ is called compact (good) whenever $X$ is compact (good).

COROLlary 5.2. There exist natural isomorphisms

$$
\boldsymbol{\omega}\left\{\mathbf{X}, \overline{\mathbf{Y}^{\mathbf{Z}}}\right\}_{c} \approx\left\{\mathbf{X}, \mathbf{Y}^{\mathbf{Z}}\right\}
$$

1) for $\mathbf{Y}$ good, $\mathbf{Z}$ compact (fixed) and $\mathbf{X}$ compact (variable);

2) for $\mathbf{Z}$ good and compact, $\mathbf{Y}$ good and arbitrary space $\mathbf{X} \in \mathfrak{P}$.

Here we have set, in analogy to $\S 4(2)$,

$$
\mathbf{Y}^{\mathbf{Z}}=\left(Y^{Z}, m-n\right),
$$

for $\mathbf{Y}=(Y, m), \mathbf{Z}=(Z, n)$; and $\mathfrak{P}^{\prime}(\ldots, \ldots)=\{\ldots, \ldots\}$.

Let $\mathbf{A}=(A, m), \mathbf{B}=(B, n)$ be in $\mathfrak{P}(A, B$ spaces, but not necessarily in Met, i.e. $A$ could be a function space). We say that $\mathbf{A}$ and $\mathbf{B}$ are of the same generalized weak homotopy type whenever there exists a chain of weak homotopy equivalences in the sense of 5.1 or of Definition 4.1 connecting $\mathbf{A}$ and $\mathbf{B}$. Recall that we write $A$ for the pair $(A, 0)$.

Corollary 5.3. If $Y$ is good and $Z$ good and compact, then the spaces $Y^{Z}$ and $D Z \wedge Y$ are of the same generalized weak homotopy type.

P r o o f. According to Theorem 4.8 we have a weak homotopy equivalence

$$
\overline{Y^{Z}} \simeq D Z \pi Y
$$

however, $\overline{Y^{Z}} \simeq Y^{Z}$ (see 5.2.2)) and $D Z \wedge Y=D Z \wedge Y$ because of $[2,1.2]$ (and by the related implication for $\mathfrak{P}$ instead of coss), observing that $D Z$ is (up to isomorphism) good and compact. 
COROLlary 5.4. For $Y$ good and compact we have a generalized weak homotopy equivalence

$$
D Y \simeq\left(S^{0}\right)^{Y} .
$$

Remarks. 1) Theorem 5.1.2) is a substitute for the assertion " $Y^{Z}$ and $\overline{Y^{Z}}$ are of the same weak homotopy type" (meaningless in view of Definition 4.1).

2) Corollary 5.4 expresses the well-known fact from classical S-duality: The S-dual of a good and compact space $Y$ (e.g. of a finite polyhedron) is of the same weak homotopy type as the "functional dual" (cf. for example [4, Theorem 3.8].) Here "weak homotopy type" has to be interpreted in our sense, but a concrete mapping (in $\mathfrak{P}$ ) inducing this equivalence in the classical way can easily be provided.

\section{References}

[1] F. W. Bauer, A strong shape theory admitting an S-dual, Topology Appl. 62 (1995), $207-232$.

[2] —, A strong shape theory with S-duality, Fund. Math. 154 (1997), 37-56.

[3] —, Duality in manifolds, Ann. Mat. Pura Appl. (4) 136 (1984), 241-302.

[4] J. M. Cohen, Stable Homotopy, Lecture Notes in Math. 165, Springer, Heidelberg, 1970 .

[5] J. Dugundji, Topology, Allyn and Bacon, Boston, 1966.

[6] B. Günther, The use of semisimplicial complexes in strong shape theory, Glas. Mat. 27 (47) (1992), 101-144.

[7] E. Spanier, Function spaces and duality, Ann. of Math. 70 (1959), 338-378.

[8] H. Thiemann, Strong shape and fibrations, Glas. Mat. 30 (50) (1995), 135-174.

Fachbereich Mathematik

Johann-Wolfgang-Goethe Universität

Robert-Mayer Str. 6-10

60054 Frankfurt a.M., Germany

E-mail: f.w.bauer@mathematik.uni-frankfurt.d400.de

Received 25 January 1996 